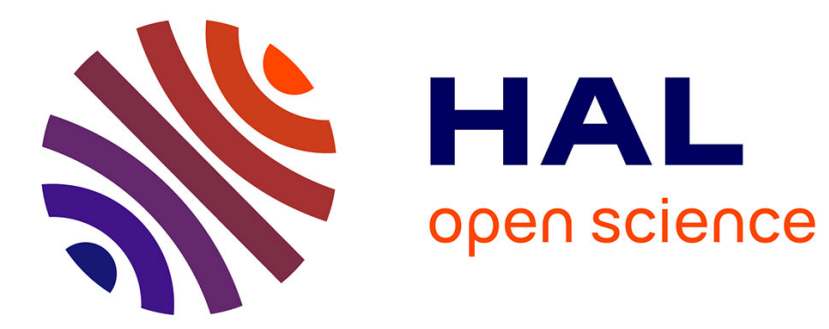

\title{
The Spiral 2 Project
}

M. Lewitowicz

\section{- To cite this version:}

M. Lewitowicz. The Spiral 2 Project. TOURS 2006, Tours Symposium on Nuclear Physics VI, Sep 2006, Tours, France. pp.91-98, 10.1063/1.2713504 . in2p3-00156691

\section{HAL Id: in2p3-00156691 https://hal.in2p3.fr/in2p3-00156691}

Submitted on 22 Jun 2007

HAL is a multi-disciplinary open access archive for the deposit and dissemination of scientific research documents, whether they are published or not. The documents may come from teaching and research institutions in France or abroad, or from public or private research centers.
L'archive ouverte pluridisciplinaire HAL, est destinée au dépôt et à la diffusion de documents scientifiques de niveau recherche, publiés ou non, émanant des établissements d'enseignement et de recherche français ou étrangers, des laboratoires publics ou privés. 


\title{
The SPIRAL 2 Project
}

\author{
Marek Lewitowicz \\ on behalf of the SPIRAL 2 project group \\ Grand Accélérateur d'Ions Lourds - GANIL \\ CEA/DSM-CNRS/IN2P3 \\ BP 55027, 14076 Caen Cedex, France
}

\begin{abstract}
The project of an important new extension of the GANIL facility - SPIRAL 2 - is shortly presented. The physics case of the facility is based on the use of high intensity stable and radioactive beams. Expected performances and main technical parameters of the facility as well as planned new experimental areas and detectors are shortly described.
\end{abstract}

Keywords: Radioactive nuclear beams, linear accelerators, mass separators, charged particle detectors, gamma arrays, neutron-induced reactions, fission, heavy-ion induced fusion, nuclear structure, nuclear reaction dynamics

PACS: 25.60.-t; 29.17.+w; 29.27.Eg; 25.85.-w

\section{INTRODUCTION - CURRENT GANIL FACILITY}

In recent years, Rare (or Radioactive) Nuclear Beams (RNBs), i.e. beams of synthesized radioactive isotopes, have been recognized by the international scientific community as one of the most promising avenues for the development of fundamental nuclear physics and astrophysics, as well as in applications of nuclear science. Through the concept of beta-beams, RNBs might also become a way to create intense beams of pure neutrinos.

The GANIL facility ${ }^{1}$ (Caen, France) is one of the major RNB and stable-ion facilities for nuclear physics, astrophysics and interdisciplinary research in Europe. Since the first beams delivered in 1983 the performances of the GANIL accelerator complex, shown in Fig. 1, was constantly improved with respect to the beam intensity, energy and available detection systems. Almost since the beginning of the experimental program, the facility delivered RNB produced "in-flight" at fragment separators like LISE and Alpha spectrometer.

More recently, in autumn 2001, the SPIRAL facility allowing for the production and post-acceleration of the ISOL-type RNB entered into operation. The facility, specialized in RNB of rare gases (He, Ne, $\mathrm{Ar}, \mathrm{Kr}$ but also $\mathrm{N}, \mathrm{O}$ and $\mathrm{F}$ ), enlarged importantly the range of experimental possibilities dedicated to study of nuclei far from stability at GANIL. In particular, low energy an in-beam gamma-ray spectroscopy, measurements of the spectroscopic factors via transfer reactions, elastic and inelastic scattering highly benefit from pure, high intensity and high optical quality RNB post-accelerated by CIME cyclotron. The production of RNB at SPIRAL 
is based on a use of high intensity beams of heavy ions impinging on a universal graphite target thus using nuclear reactions in inverse kinematics.

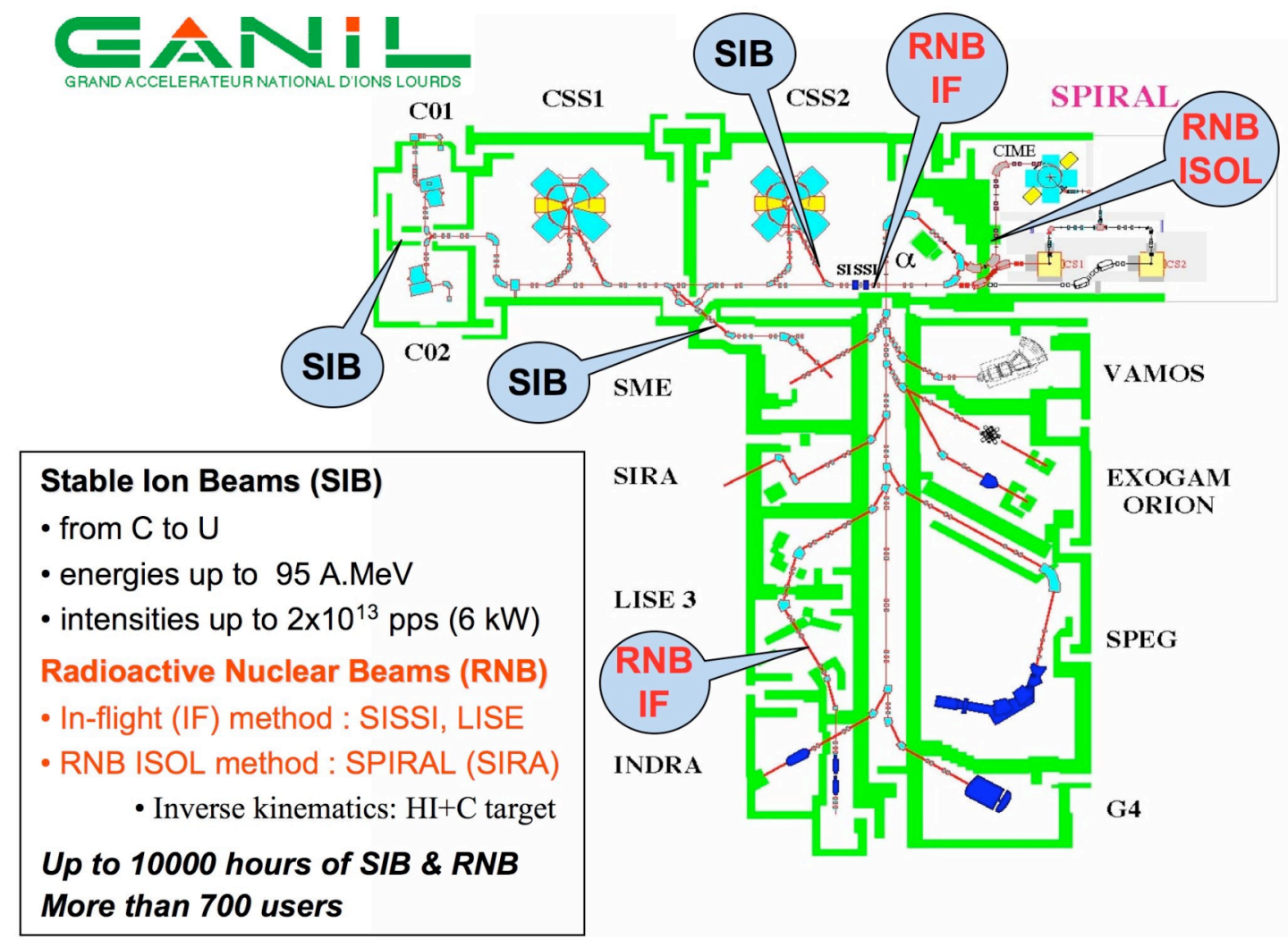

FIGURE 1. Layout of the current GANIL.SPIRAL facility and its main characteristics.

However, the available intensities and energies of the GANIL's stable-ion beams (see fig. 1) limit the use of high intensity RNB to relatively light nuclei $(A<80)$.

Since the beginning of the SPIRAL project it was proposed to enlarge the range of accelerated ions by production of high intensity RNB of fission fragments. This idea, after several years of discussions and an important preliminary study phase led, in particular, in the framework of the European RTT Program was concretized in the SPIRAL 2 project ${ }^{2}$.

The project is following the European road map for RNB facilities defined by NuPECC $^{3}$ (Nuclear Physics European Collaboration Committee - an expert committee of the European Science Foundation), which recommend the construction of two complementary next-generation RIB facilities in Europe. One is based on in-flight fragmentation (IF) as proposed for the FAIR facility at GSI (Darmstadt, Germany) and the other on the isotope-separation on-line (ISOL) method, largely developed at the CERN-ISOLDE facility over the last thirty years (the EURISOL project ${ }^{6}$ ). However, a full engineering design study and necessary R\&D programme mean that the expected beginning of operation of the EURISOL facility will only be around 2020. Because of the time-line for EURISOL as well as of important unsolved yet technological issues, NuPECC recommends the construction of intermediate-generation facilities that will 
benefit the EURISOL project in terms of R\&D and that will give the community opportunities to perform research and develop applications with RNB. Among the proposed intermediate facilities, SPIRAL2 meets the criteria of European dimension in terms of physics potential, site and size of the investment.

\section{LAYOUT AND PERFORMANCES OF THE SPIRAL 2 FACILITY}

The SPIRAL 2 facility (fig. 2) is based on a high-power, superconducting driver LINAC, which will deliver a high-intensity, $40 \mathrm{MeV}$ deuteron beam as well as a variety of heavy-ion beams with mass-to-charge ratio of 3 and energy up to 14.5 $\mathrm{MeV} /$ nucleon. Using a carbon converter, the $5 \mathrm{~mA}$ deuteron beam and a uranium carbide target, fast-neutron induced fission is expected to reach a rate of up to $10^{14}$ fissions/s. The RNB intensities in the mass range from $A=60$ to $A=140$ will be of the order of $10^{6}$ to $10^{11}$ particles/s (pps) surpassing by one or two order of magnitude any existing facilities in the world. For example, the intensities should reach $10^{9} \mathrm{pps}$ for ${ }^{132} \mathrm{Sn}$ and $10^{10} \mathrm{pps}$ for ${ }^{92} \mathrm{Kr}$. A direct irradiation of the $\mathrm{UC}_{2}$ target with beams of deuterons, ${ }^{3,4} \mathrm{He},{ }^{6,7} \mathrm{Li}$, or ${ }^{12} \mathrm{C}$ can be used if higher excitation energy leads to higher production rate for a specific nucleus of interest.

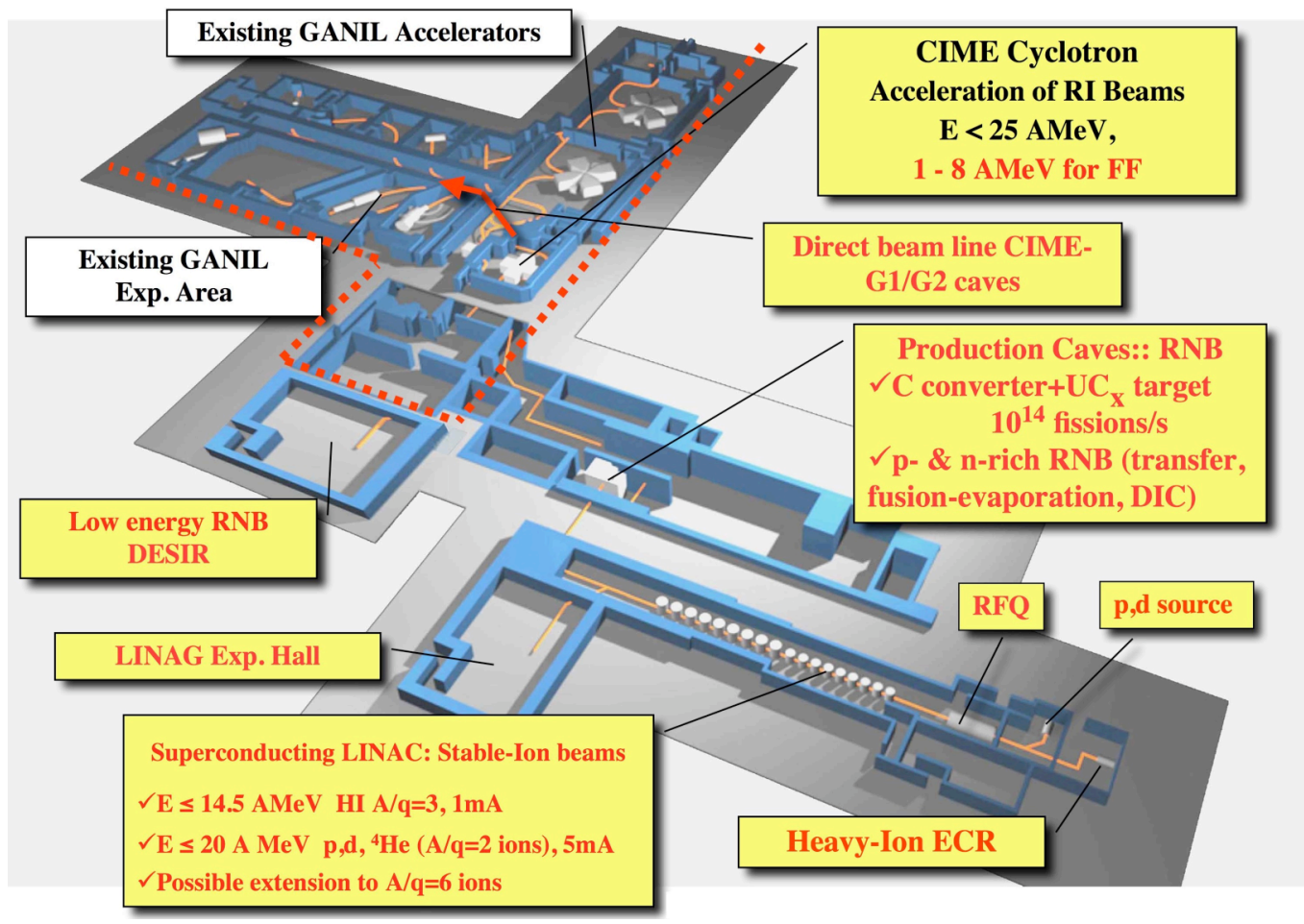

FIGURE 2. Layout of the SPIRAL 2 facility and its main characteristics. The dotted line corresponds to the separation between the current (upper part) and new facilities.

These neutron-rich fission RNB could be complemented by beams of nuclei near the proton drip-line, provided by fusion-evaporation or transfer reactions. For example, a production of up to $8 \times 10^{4}$ atoms of ${ }^{80} \mathrm{Zr}$ per second using a $200 \mu \mathrm{A}$ 
${ }^{24} \mathrm{Mg}^{8+}$ beam on a ${ }^{58} \mathrm{Ni}$ target should be possible. Similarly, the heavy- and light-ion beams from LINAG can also be used directly on different production targets to produce high-intensity light RNB with the ISOL technique.

The extracted $1+$ radioactive ions will be subsequently injected to the $1+/ \mathrm{n}+$ charge booster (ECR ion source) and post-accelerated to energies of up to 20 $\mathrm{MeV} /$ nucleon (typically 6-7 MeV/nucleon for fission fragments) by the existing CIME cyclotron.

Thus, using several different production mechanisms and techniques, SPIRAL 2 would allow users to perform experiments with a wide range of neutron- and protonrich nuclei far from the line of stability.

One of the important features of the future GANIL/SPIRAL 1/SPIRAL 2 facility will be the possibility to deliver up to five stable or radioactive beams to different users simultaneously in the energy range from $\mathrm{keV}$ to several tens of $\mathrm{MeV} /$ nucleon.

\section{SCIENTIFIC OPPORTUNITIES AT SPIRAL 2}

In the following paragraphs, only several examples of a rich and multipurpose scientific program proposed at SPIRAL 2 will be discussed. More complete presentation of the scientific case of SPIRAL 2 can be found in the recently accomplish White Book of SPIRAL 2 (see ref. 4).

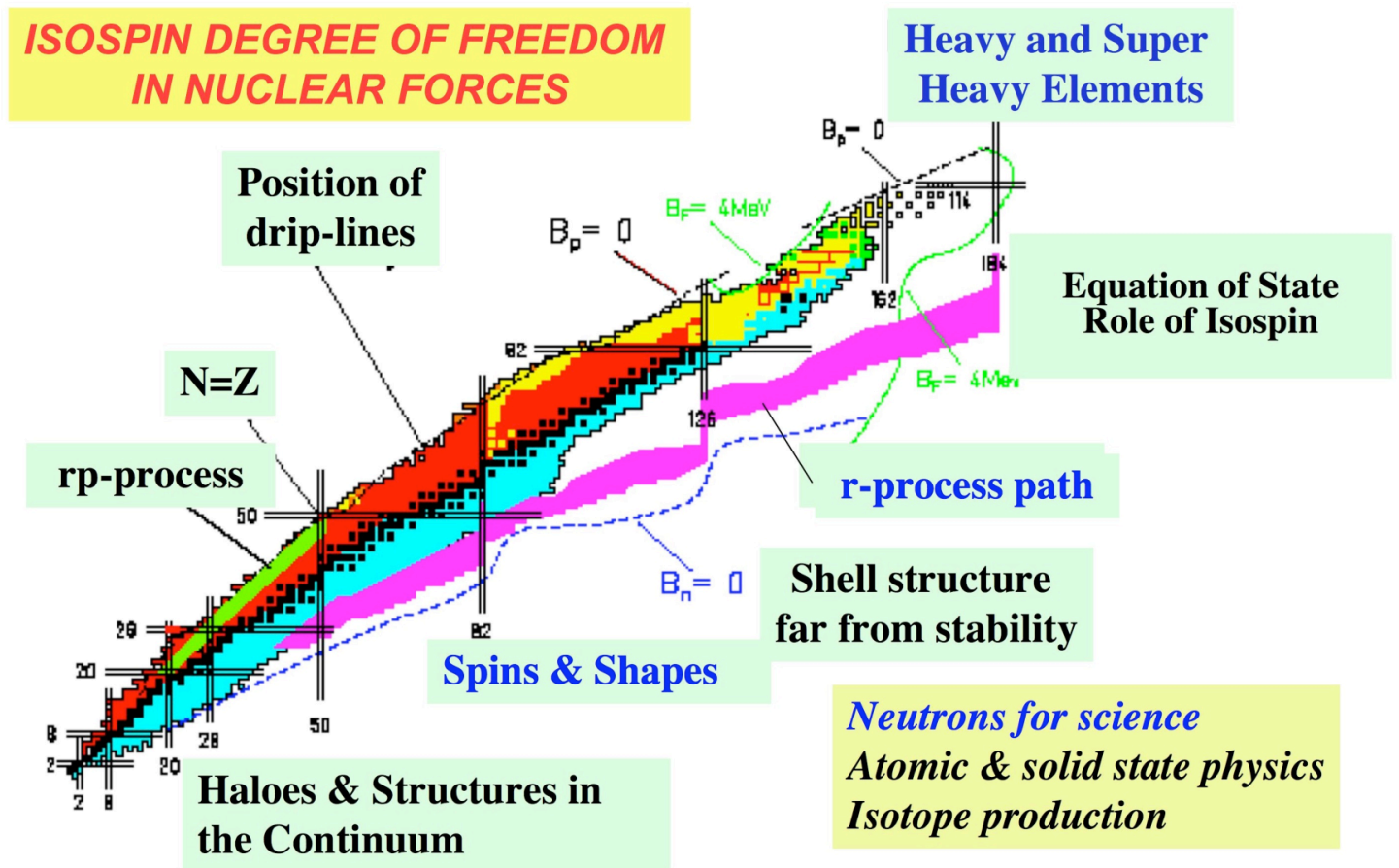

FIGURE 3. Main topics of the scientific case of SPIRAL 2. 


\section{Physics Of Nuclei Far From Stability}

A major part of the experimental and theoretical research program at the SPIRAL 2 facility will follow the fundamental motivation of basic nuclear science research, which is trying to establish a bridge between the nucleon-nucleon inside a nucleus and the underlying quarks and gluons. The modifications induced by the nuclear medium make the problem extremely complex. The result is that, although the reactions between nucleons in the vacuum have been studied in detail, the forces, which bind the nucleons in the nucleus, are neither well known nor really understood. However, setting up the interaction between its constituents is not enough to understand the properties of a complex object like the nucleus. One should also determine the organization of the nucleons and the phenomena at the origin of the observed structures.

This research on the nucleus and on the interactions between its constituents progresses using nuclei with unusual neutron-to-proton ratios, artificially produced in laboratories, which open up a considerable field of investigation. By allowing the study of very varied situations, the nuclei far from stability highlight the phenomena at the origin of the cohesion of the nucleus. SPIRAL 2 thanks to very high intensities of RNB will thus give access to a whole range of experiments (from elastic scattering to fusion-evaporation reactions), which are inaccessible today.

New exotic shapes and excitation modes, e.g. halo-like and molecular structures, and new modes of nuclear decay have been recently observed, while tests of fundamental symmetries, testing and refinement of the Standard Model of fundamental interactions, and exploration of the 'magic' numbers of protons and nuclei in very exotic nuclei are all enticing avenues of discovery.

\section{Nucleosynthesis}

Nuclei were created during major events during the evolution of the Universe and are the testimony of the origin of matter: while the lightest elements were created in the first minutes following the Big Bang, heavier ones are synthesized in stars. However, these various nucleosynthesis processes involve radioactive nuclei. Due to their very short lifetimes, most of them have not survived long enough to be present on earth. To be studied these "exotic" nuclei must therefore be synthesized in laboratories. SPIRAL 2 will produce abundantly nuclei lying on or in a close proximity to the $r$ and rp-process paths, opening up new fields of investigation. In particular, a new insight on nucleosynthesis might be achieved using transfer reactions $(\mathrm{d}, \mathrm{p}),(\mathrm{p}, \mathrm{d})$, etc. The production of radioactive targets in order to measure neutron capture cross-sections might help to elucidate several important problems in the understanding of the s-process.

\section{Neutrons For Science}

One of the interesting possibilities, which will open with the SPIRAL 2 facility is related to the production of a high neutron flux in the energy range from several hundreds of $\mathrm{keV}$ up to about $40 \mathrm{MeV}$ (fig. 4). Thanks to that, the facility will offer a 
unique opportunity for material irradiations and cross-section measurements, both for fission-related (notably accelerator driven systems (ADS) and Gen-IV fast reactors) and fusion-related research.
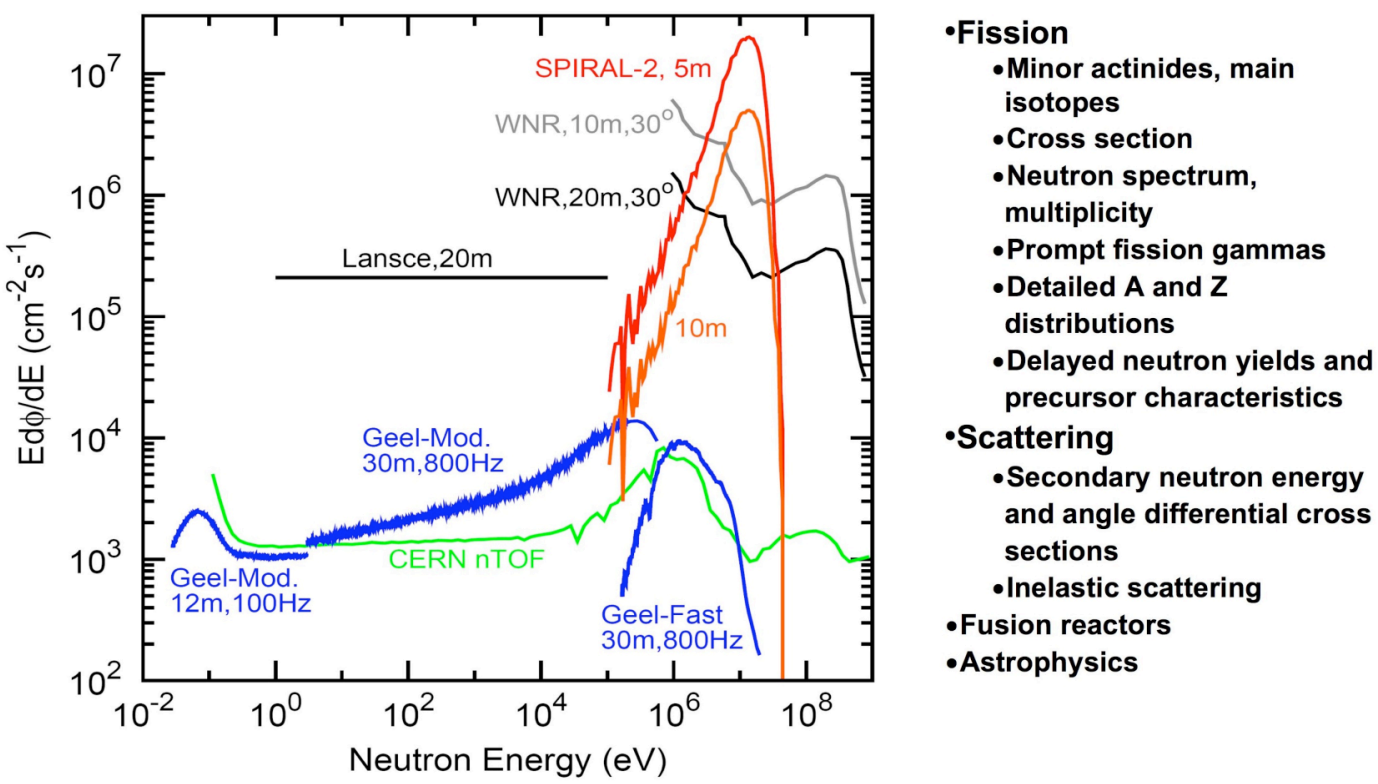

FIGURE 4. Neutron flux, which might be achieved at the "n-tof" facility at SPIRAL 2 compared to the existing facilities. A list of topics relevant to the scientific program at the proposed facility is shown.

The above experimental data require specific facilities like pulsed neutron beams for cross-section measurements by time-of-flight or dedicated irradiation stations for activation analysis. The high neutron fluxes with high and variable energy spectra available at SPIRAL 2 are very attractive to perform the measurements of the transmutation-incineration of nuclear waste and minor actinides in particular. The high neutron fluxes would allow the measurements of small reaction cross-sections and/or with very small targets, which might be rare, expensive, and in some cases radioactive. The energy range and conditions offered by SPIRAL 2 time-of-flight facility is complementary to other such facilities in Europe, notably GELINA of the European Commission's Joint Research Centre in Geel and the CERN based n_TOF facility. For example, the design of IFMIF facility requires reliable data up to $55 \mathrm{MeV}$ neutron energy. In this case, the neutron spectrum will be determined by the activation of dosimetry foils, and for most of the suitable reactions the cross-sections have been measured once with unacceptable uncertainty or never at all and the evaluated data files are in disagreement. A lot of cross section measurements can be performed by activation technique, and the SPIRAL 2 fluxes are particularly well adapted for fast neutron measurements. Cross-sections of deuteron-induced reactions on structural materials are also needed, and a deuteron activation station in the converter cave could ensure such measurements up to $40 \mathrm{MeV}$. More details on this topic can be found in ref. 5. 


\section{NEW EXPERIMENTAL AREAS AND NEW DETECTORS FOR SPIRAL 2}

In the framework of the SPIRAL 2 project two new experimental areas will be constructed. One dedicated to the experiments with high intensity stable beams delivered by LINAG and one devoted to research program with low energy RNB proposed recently by the DESIR collaboration (fig. 5).

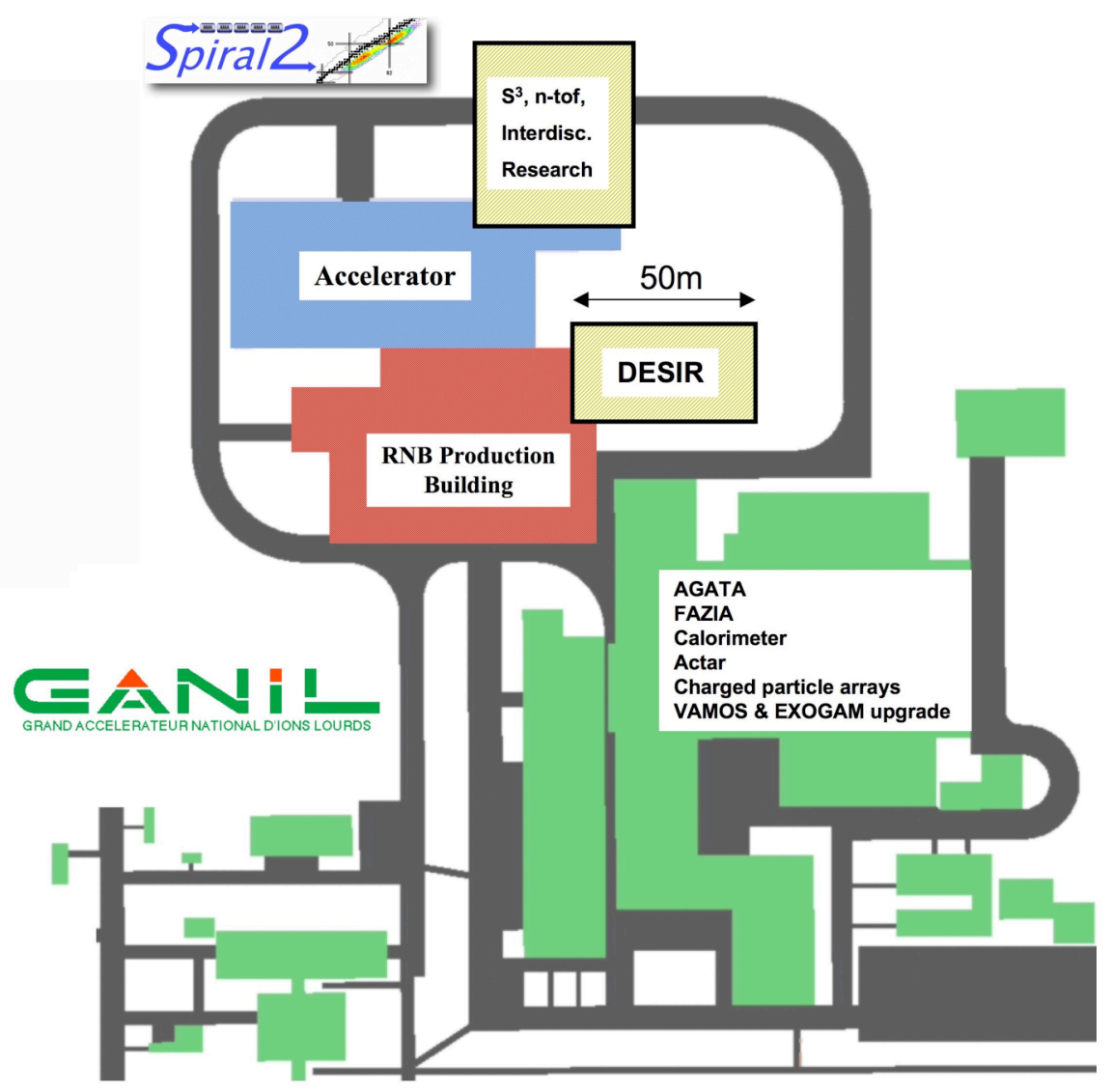

FIGURE 5. A preliminary layout of the GANIL/SPIRAL 1/SPIRAL 2 facility. Two new experimental areas (with contours) are shown and some of the proposed new detectors are listed.

Relatively moderate intensities and high cost of radioactive beams impose a use of the most efficient and innovative detection systems such as the magnetic spectrometer VAMOS, the $4 \Pi$ gamma-array EXOGAM and AGATA as well as charged particle detectors like MAYA, MUST 2 and TIARA. Several new concepts of the detection systems (DESIR, FAZIA, SPAGA) and a new separator/spectrometer $\mathrm{S}^{3}$ located in dedicated experimental halls are currently under consideration. Most of the existing detection systems and the existing experimental area should be adopted to take a full benefit of the high intensity (up to $10^{11} \mathrm{pps}$ ) RNB.

A process leading to the definition of the detectors and corresponding collaborations was initiated recently via call for letters of intent for SPIRAL 2 (see ref. 4 for details). The evaluation of the letters of intent by the SPIRAL 2 Scientific 
Advisory Committee took place in the end of October 2006. It is foreseen that a call for full proposals will be launched in 2007 and signatures of Memorandums of Understanding related to the construction of new equipment will take place in 20082009.

\section{CONCLUSIONS}

On May 23, 2005, the French government approved the construction of SPIRAL 2 a new $130 \mathrm{M} €$ RNB facility at GANIL. Its construction cost is shared by the French funding agencies CNRS/IN2P3 and CEA/DSM, the regional authorities of BasseNormandie and international partners. The first beams are expected at SPIRAL 2 by 2011-2012. The project management group responsible for the construction phase was formed recently. Several agreements with international partners were signed recently. The construction of the SPIRAL 2 driver (LINAG) just started. The detailed definition of the RNB production building, of the experimental areas and of the dedicated detectors is entering in the decisive phase.

SPIRAL 2 will reinforce European leadership in the field of exotic nuclei and will serve a community of about 600 scientists.

\section{REFERENCES}

1. www.ganil.fr

2. www.ganil.fr/research/developments/spiral2/origin.html.

3. www.nupecc.org

4. www.ganil.fr/research/developments/spiral2/index.html

5. X. Ledoux et al., Neutrons for Science, SPIRAL 2 Letter of Intent No. 17, www.ganil.fr/research/developments/spiral2/loi texts.html

6. www.eurisol.org 